\title{
Chapter 40 \\ Report on Global Environment \\ Competitiveness of Costa Rica
}

Costa Rica is a country in Central America, bordered by Nicaragua to the north, Panama to the southeast, the Pacific Ocean to the west, and the Caribbean Sea to the east. It covers 51.1 thousand of square kilometers and has a population of 4.73 million. Its GDP reaches $\$ 40.87$ billion in 2011 . Through the index system and evaluation

Score: 57.20

Rank: 7 model, the environment competitiveness index of Costa Rica ranks at 7 in 133 countries.

Fig. 40.1 Contribution of sub-index to GEC

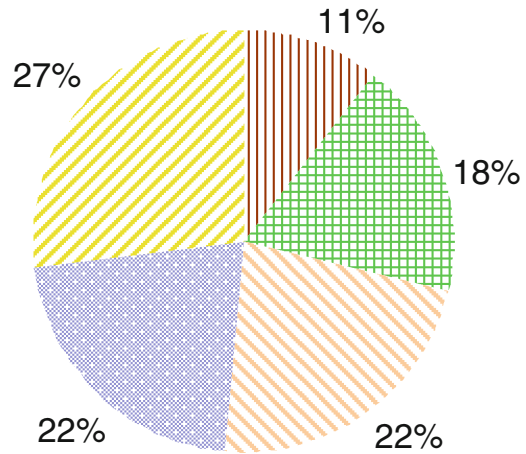

III Resource Environment Competitiveness

* Ecological Environment Competitiveness Environment Carrying Competitiveness

Environment Management Competitiveness Environment Harmony Competitiveness 


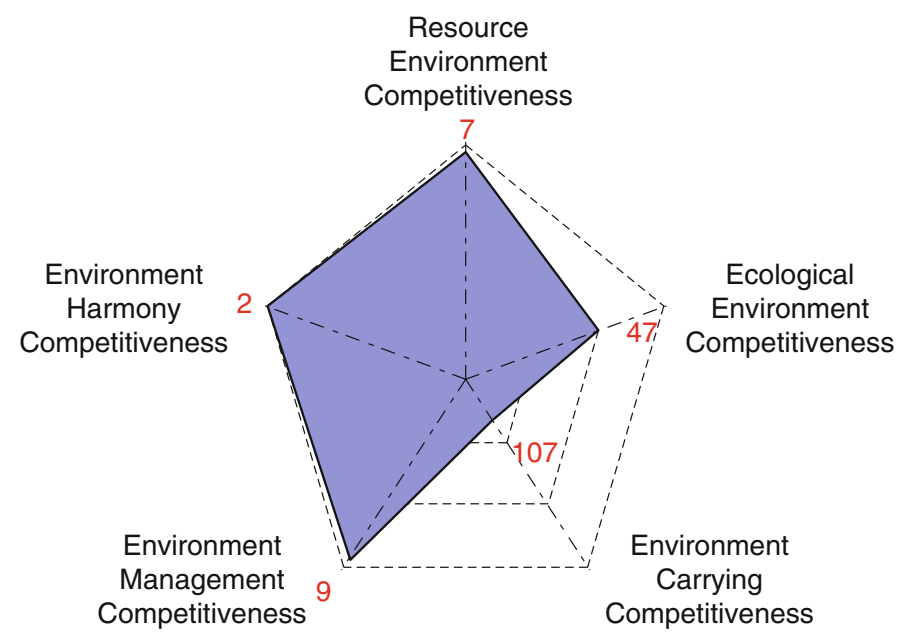

Fig. 40.2 Rank of sub-index of GEC

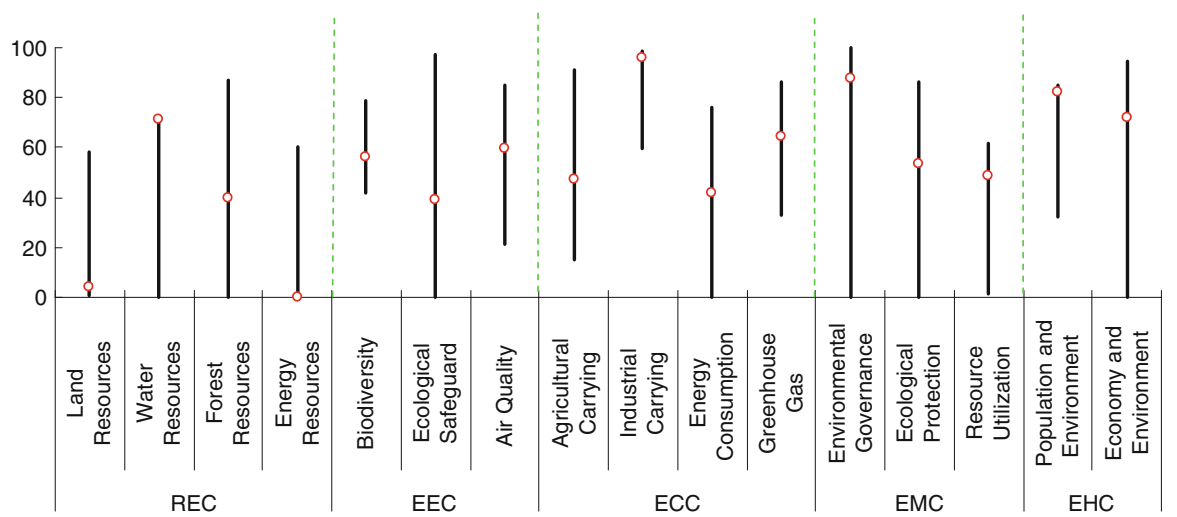

Fig. 40.3 Score and rank of the pillars of GEC

Table 40.1 Score and rank of all indicators of GEC

\begin{tabular}{|c|c|c|c|c|c|}
\hline Indicators & Score & Rank & Indicators & Score & Rank \\
\hline 1 Resource Environment & 30.08 & 7 & Groundwater & 100.00 & 1 \\
\hline Competitiveness & & & \multirow{2}{*}{$\begin{array}{l}\text { Total internal renewable } \\
\text { water resources }\end{array}$} & \multirow[t]{2}{*}{100.00} & \multirow[t]{2}{*}{1} \\
\hline 1.1 Land Resources & 3.84 & 125 & & & \\
\hline Land area per capita & 1.92 & 80 & 1.3 Forest Resources & 39.52 & 27 \\
\hline $\begin{array}{l}\text { Percentage of arable land to } \\
\text { total land area }\end{array}$ & 7.86 & 104 & $\begin{array}{l}\text { Growing stock in forest and } \\
\text { other wooded land }\end{array}$ & 50.80 & 76 \\
\hline Arable land per capita & 2.39 & 117 & \multirow{2}{*}{$\begin{array}{l}\text { Proportion of land area } \\
\text { covered by forest }\end{array}$} & \multirow[t]{2}{*}{57.87} & \multirow[t]{2}{*}{18} \\
\hline 1.2 Water Resources & 71.34 & 1 & & & \\
\hline Surface water & 15.83 & 16 & Forest area per capita & 3.78 & 47 \\
\hline Annual precipitation & 69.55 & 11 & 1.4 Energy Resources & 0.00 & 125 \\
\hline
\end{tabular}


Table 40.1 (continued)

\begin{tabular}{|c|c|c|c|c|c|}
\hline Indicators & Score & Rank & Indicators & Score & Rank \\
\hline Fossil energy & 0.00 & 64 & \multirow{2}{*}{$\begin{array}{l}\text { Energy consumption per unit } \\
\text { of land area }\end{array}$} & \multirow[t]{2}{*}{99.81} & \multirow[t]{2}{*}{61} \\
\hline Energy production & 0.51 & 82 & & & \\
\hline $\begin{array}{l}\text { Proportion of combustible } \\
\text { renewable and waste to }\end{array}$ & 18.26 & 45 & $\begin{array}{l}\text { Ratio of clean energy } \\
\text { consumption }\end{array}$ & 42.71 & 13 \\
\hline total energy consumption & & & Elasticity of energy & 14.05 & 52 \\
\hline $\begin{array}{l}\text { Net energy imports of the } \\
\text { energy consumption }\end{array}$ & 6.48 & 84 & $\begin{array}{l}\text { Elasticity of electric power } \\
\text { consumption }\end{array}$ & 11.06 & \multirow[t]{2}{*}{47} \\
\hline $\begin{array}{l}2 \text { Ecological Environment } \\
\text { Competitiveness }\end{array}$ & 52.46 & 47 & $\begin{array}{l}\text { consumption } \\
\text { 3.4 Greenhouse Gas }\end{array}$ & 64.19 & \\
\hline $\begin{array}{l}2.1 \text { Biodiversity } \\
\text { Threatened fish species }\end{array}$ & $\begin{array}{l}56.46 \\
76.42\end{array}$ & $\begin{array}{r}93 \\
105\end{array}$ & $\begin{array}{l}\text { Growth rate of } \mathrm{CO}_{2} \\
\text { emissions }\end{array}$ & 51.06 & 71 \\
\hline Threatened mammal species & 95.11 & 48 & $\begin{array}{l}\text { Growth rate of Methane } \\
\text { emissions }\end{array}$ & 54.12 & 92 \\
\hline $\begin{array}{l}\text { Threatened plant species } \\
\text { GEF benefits index for } \\
\text { biodiversity }\end{array}$ & $\begin{array}{r}93.17 \\
8.80\end{array}$ & $\begin{array}{r}108 \\
30\end{array}$ & $\begin{array}{l}\text { emissions } \\
\mathrm{CO}_{2} \text { emissions per unit of } \\
\text { land area }\end{array}$ & 99.86 & 58 \\
\hline 2.2 Ecological Safeguard & $\begin{array}{l}38.78 \\
55.16\end{array}$ & 24 & $\begin{array}{l}\mathrm{CO}_{2} \text { emissions per unit of } \\
\text { energy consumption }\end{array}$ & 63.59 & 30 \\
\hline Marine protected areas & 14.21 & 20 & $\begin{array}{l}4 \text { Environment Management } \\
\text { Competitiveness }\end{array}$ & 62.37 & 9 \\
\hline $\begin{array}{l}\text { 2.3 Air Quality } \\
\text { Inhalable particles (PM10) }\end{array}$ & $\begin{array}{l}59.73 \\
80.29\end{array}$ & $\begin{array}{l}62 \\
57\end{array}$ & \multirow{3}{*}{$\begin{array}{l}\text { 4.1 Environmental } \\
\text { Governance } \\
\text { Agricultural chemicals } \\
\text { regulation }\end{array}$} & 87.78 & 72 \\
\hline Particulate matter (PM2.5) & 94.70 & 14 & & 76.19 & 61 \\
\hline Index of indoor air pollution & 27.60 & 66 & & 10.19 & 01 \\
\hline Nitrogen oxides emission & 68.72 & 28 & \multirow{3}{*}{$\begin{array}{l}\text { Percentage of the rural } \\
\text { population with access } \\
\text { to an improved water } \\
\text { source }\end{array}$} & \multirow[t]{3}{*}{91.00} & \multirow[t]{3}{*}{58} \\
\hline Sulfur dioxide emission & 40.95 & 16 & & & \\
\hline $\begin{array}{l}3 \text { Environment Carrying } \\
\text { Competitiveness }\end{array}$ & 64.13 & 107 & & & \\
\hline 3.1 Agricultural Carrying & 47.02 & 128 & \multirow{3}{*}{$\begin{array}{l}\text { Percentage of the urban } \\
\text { population with access } \\
\text { to an improved water } \\
\text { source }\end{array}$} & \multirow[t]{3}{*}{100.00} & \multirow[t]{3}{*}{1} \\
\hline $\begin{array}{l}\text { Cereal yield per unit of } \\
\text { arable land }\end{array}$ & 37.16 & 48 & & & \\
\hline $\begin{array}{l}\text { Fertilizer consumption per } \\
\text { unit of arable land }\end{array}$ & 16.43 & 127 & & & \\
\hline $\begin{array}{l}\text { unit of arable land } \\
\text { Annual freshwater } \\
\text { withdrawals for }\end{array}$ & 90.76 & 108 & $\begin{array}{l}\text { 4.2 Ecological Protection } \\
\text { Area of plantation and } \\
\text { afforestation }\end{array}$ & $\begin{array}{r}53.36 \\
0.31\end{array}$ & $\begin{array}{l}12 \\
64\end{array}$ \\
\hline agriculture per unit of & & & Biome protect & 91.90 & 21 \\
\hline $\begin{array}{l}\text { arable land } \\
\text { 3.2 Industrial Carrying }\end{array}$ & 96.03 & 8 & $\begin{array}{l}\text { Overfishing of fishing } \\
\text { resources }\end{array}$ & 85.56 & 4 \\
\hline $\begin{array}{l}\text { Net exports of goods as a } \\
\text { percentage of GDP }\end{array}$ & 84.19 & 49 & $\begin{array}{l}\text { 4.3 Resource Utilization } \\
\text { Utilization rate of water }\end{array}$ & $\begin{array}{r}48.97 \\
0.08\end{array}$ & $\begin{array}{l}15 \\
93\end{array}$ \\
\hline Electric power consumption & 99.95 & 3 & $\begin{array}{l}\text { Utilization rate of water } \\
\text { resources }\end{array}$ & & 93 \\
\hline $\begin{array}{l}\text { per unit of value added } \\
\text { of industry }\end{array}$ & & & $\begin{array}{l}\text { Percentage of total internal } \\
\text { renewable water }\end{array}$ & 100.00 & 1 \\
\hline $\begin{array}{l}\mathrm{SO}_{2} \text { emissions per unit of } \\
\text { value added of industry }\end{array}$ & 99.99 & 6 & $\begin{array}{l}\text { resources to total water } \\
\text { resources }\end{array}$ & & \\
\hline $\begin{array}{l}\text { Annual freshwater } \\
\text { withdrawals for industry }\end{array}$ & 99.98 & 4 & $\begin{array}{l}\text { Percentage of agricultural } \\
\text { land to total land area }\end{array}$ & 41.35 & 85 \\
\hline $\begin{array}{l}\text { per value added of } \\
\text { industry }\end{array}$ & & & $\begin{array}{l}\text { Percentage of fossil fuel } \\
\text { energy consumption to }\end{array}$ & 54.44 & 28 \\
\hline 3.3 Energy Consumption & 41.91 & 20 & total energy consumption & & \\
\hline
\end{tabular}


Table 40.1 (continued)

\begin{tabular}{lcclcr}
\hline Indicators & Score & Rank & Indicators & Score & Rank \\
\hline $\begin{array}{l}5 \text { Environment Harmony } \\
\text { Competitiveness }\end{array}$ & 76.97 & 2 & $\begin{array}{l}\mathrm{CO}_{2} \text { emissions per capita } \\
\text { Energy consumption per } \\
\text { capita }\end{array}$ & 96.27 & 43 \\
$\begin{array}{l}5.1 \text { Population and } \\
\text { Environment }\end{array}$ & 82.06 & 8 & $\begin{array}{l}\text { 5.2 Economy and } \\
\text { Environment }\end{array}$ & 71.88 & 18 \\
$\begin{array}{c}\text { Percentage of population } \\
\text { with access to Improved } \\
\text { sanitation facilities }\end{array}$ & 95.00 & 46 & $\begin{array}{c}\text { Land resource utilization } \\
\text { efficiency }\end{array}$ & 0.23 & 42 \\
$\begin{array}{c}\text { Motor vehicles per 1,000 } \\
\text { people }\end{array}$ & 79.75 & 77 & $\begin{array}{c}\text { Sulfur dioxide emissions per } \\
\text { unit of GDP }\end{array}$ & 98.42 & 22 \\
$\begin{array}{c}\text { Renewable internal } \\
\text { freshwater resources per } \\
\text { capita }\end{array}$ & 26.75 & 15 & $\begin{array}{c}\text { Carbon dioxide emissions } \\
\text { per unit of GDP }\end{array}$ & 95.40 & 10 \\
$\mathrm{SO}_{2}$ emissions per capita & 98.31 & 31 & $\begin{array}{c}\text { Energy consumption per unit } \\
\text { of GDP }\end{array}$ & 93.48 & 25 \\
\hline
\end{tabular}

Table 40.2 Rank distribution of the individual indicators of GEC

\begin{tabular}{|c|c|c|c|c|c|c|}
\hline Sub-index & $\begin{array}{l}\text { Number of } \\
\text { the individual } \\
\text { indicators }\end{array}$ & $\begin{array}{l}\text { Rank } \\
1-10\end{array}$ & $\begin{array}{l}\text { Rank } \\
11-30\end{array}$ & $\begin{array}{l}\text { Rank } \\
31-60\end{array}$ & $\begin{array}{l}\text { Rank } \\
61-100\end{array}$ & $\begin{array}{l}\text { Rank } \\
101-133\end{array}$ \\
\hline $\begin{array}{l}\text { Resource Environment } \\
\text { Competitiveness }\end{array}$ & 14 & 2 & 4 & 1 & 4 & 3 \\
\hline $\begin{array}{l}\text { Ecological Environment } \\
\text { Competitiveness }\end{array}$ & 11 & 0 & 4 & 2 & 3 & 2 \\
\hline $\begin{array}{l}\text { Environment Carrying } \\
\text { Competitiveness }\end{array}$ & 15 & 3 & 2 & 5 & 3 & 2 \\
\hline $\begin{array}{l}\text { Environment Management } \\
\text { Competitiveness }\end{array}$ & 10 & 1 & 3 & 1 & 5 & 0 \\
\hline $\begin{array}{l}\text { Environment Harmony } \\
\text { Competitiveness }\end{array}$ & 10 & 2 & 3 & 4 & 1 & 0 \\
\hline Total & 60 & 8 & 16 & 13 & 16 & 7 \\
\hline
\end{tabular}

Open Access This chapter is distributed under the terms of the Creative Commons Attribution Noncommercial License, which permits any noncommercial use, distribution, and reproduction in any medium, provided the original author(s) and source are credited. 